Revue d'histoire de l'enfance « irrégulière »

Le Temps de l'histoire

11 | 2009

Paroles libres, paroles captives

\title{
Entre expériences des enfants et écrits institutionnels : des histoires de placement
}

Emilie Potin

\section{(2) OpenEdition \\ 12 Journals}

Édition électronique

URL : http://journals.openedition.org/rhei/3067

DOI : 10.4000/rhei.3067

ISBN : 978-2-7535-1650-2

ISSN : 1777-540X

Éditeur

Presses universitaires de Rennes

Édition imprimée

Date de publication : 1 octobre 2009

ISBN : 978-2-7535-0927-6

ISSN : 1287-2431

Référence électronique

Emilie Potin, "Entre expériences des enfants et écrits institutionnels : des histoires de placement », Revue d'histoire de l'enfance « irrégulière » [En ligne], 11 | 2009, mis en ligne le 01 octobre 2011, consulté le 04 décembre 2020. URL : http://journals.openedition.org/rhei/3067 ; DOI : https://doi.org/10.4000/ rhei.3067

Ce document a été généré automatiquement le 4 décembre 2020.

(c) PUR 


\title{
Entre expériences des enfants et écrits institutionnels : des histoires de placement
}

\author{
Emilie Potin
}

1 Dans les bureaux de l'Aide sociale à l'enfance, la classothèque consigne les dossiers de placement d'un enfant, d'une fratrie, d'une famille. Pas de noms mais des numéros: $33483,17850 \ldots$ Un numéro pour accéder à des écrits, des écrits pour retracer une histoire de placement. Mais est-ce que ces écrits suffisent à comprendre chaque histoire ? Que nous disent les écrits et qu'est-ce qu'ils nous taisent de l'expérience de placement? La question centrale que nous proposons de traiter ici est celle de la mémoire du placement ; celle des écrits, celle des acteurs. Au travers de ce qui est dit et de ce qui est écrit, il s'agit de reconstruire les logiques en jeu, les manières de donner un sens à l'histoire du placement et de donner, pour les acteurs familiaux (enfant et parents) du sens à leur histoire.

2 Entre ce qui est écrit au moment de la séparation physique ${ }^{1}$, ce qui est compris par les uns et les autres et ce qui est raconté par l'enfant, par les parents, par les professionnels de la protection de l'enfance, se situe probablement l'histoire du placement dans toute sa complexité, c'est-à-dire comme le résultat socialement construit de points de vue individuels et collectifs, de normes et de valeurs, d'histoires individuelles, familiales et institutionnelles, etc.

3 Cet article s'appuie sur les matériaux recueillis à l'occasion d'une étude sociologique commandée par le conseil général du Finistère sur les parcours de prise en charge institutionnelle des enfants qui lui sont confiés ${ }^{2}$. Le travail d'enquête a été réalisé en deux temps: l'examen, dans un premier temps, de 350 dossiers de l'Aide sociale à l'enfance ; puis la réalisation d'entretiens ${ }^{3}$ auprès d'une cinquantaine d'acteurs (jeunes, parents et professionnels du champ de la protection de l'enfance). Le corpus documentaire comprend $1 / 6^{\text {ème }}$ des placements administratifs et judiciaires en cours de prise en charge en juin 2006 (début de l'enquête) et des placements ayant pris fin au cours de l'année 2005. Pour les parcours les plus longs et les plus précoces, les dossiers 
ont été ouverts à partir de 1987 et, pour les plus récents, en 2006. Notre enquête fait apparaître trois types de parcours qui reprennent des éléments objectifs (notamment la durée du placement, le nombre de déplacements, l'âge de l'enfant au moment du premier placement...) et des éléments plus subjectifs qui relèvent de l'expérience des acteurs, de ce qu'ils en disent. Nous les avons appelés : les "enfants placés», les "enfants déplacés ", les "enfants re-placés».

Nous avons travaillé à partir de matériaux du présent, c'est-à-dire d'archives ${ }^{4}$ toujours alimentées au moment de l'enquête. Il s'agit de les appréhender ici au travers des éléments de connaissance qu'elles apportent à l'enquête concernant la "petite " histoire, les parcours de vie mais également de s'y intéresser en tant qu'objet de recherche au travers du mode de construction de ces écrits qui constitueront les témoins de l'histoire collective des placements.

5 Ces dossiers s'inscrivent sur deux décennies où le système de la protection de l'enfance a connu des mutations importantes d'un point de vue organisationnel, politique et législatif. Un double mouvement ${ }^{5} s^{\prime}$ 'est mis en place : à la fois une internationalisation des références autour de la protection de l'enfance (Convention internationale des droits de l'enfant) et une décentralisation française de la mise en œuvre des politiques à l'échelle départementale. Les matériaux sont également les témoins de la période qui a préparé la loi du 5 mars 2007 et de celle qui précède sa mise en application. Ce contexte historique est le terreau à partir duquel prennent place les pratiques et expériences que nous allons décrire. "L'historicité de l'objet est le principe de réalité de la sociologie. Le sociologue ne reste sociologue que dans la mesure où il est sans cesse rappelé à l'ordre historique. $»^{6}$

Cet article se propose de définir les contours des histoires de placement à partir de la composition des dossiers (qui est regardé ? Sous quels aspects? Qui œuvre pour leur composition?) et à partir des expériences des enfants liées à leurs parcours de placement.

\section{Le sens du placement, l'essence du placement}

$7 \quad$ Les écrits constituent la trace de l'intervention dans le cadre du placement. Ils éclairent les motifs de la décision prise en amont et justifient tout au long de la prise en charge la poursuite du placement. Ces dossiers sont un instrument pour accéder à l'histoire, ils disent ce que l'institution qui les consigne souhaite garder et témoignent au-delà des règles formelles concernant l'archivage de la particularité de chaque placement par la taille du dossier, par des histoires singulières, par des échanges de courrier...

8 La pièce maîtresse de chaque dossier de placement est le rapport social ou les rapports sociaux ; y sont consignés l'histoire du placement et l'histoire familiale ${ }^{7}$, l'évolution de l'enfant dans son lieu d'accueil et dans ses activités scolaires et sociales, la dynamique des liens familiaux avec la famille d'origine, les préconisations autour de la poursuite ou de la levée du placement. En interrogeant le parcours de placement de l'enfant, nous avons regardé ces dossiers au travers de deux dynamiques: la dynamique des déplacements dans le temps et dans l'espace et la dynamique sociale, autrement dit le passage d'un groupe à l'autre, du milieu d'origine vers le milieu d'accueil. Nous avons cependant été contraints de limiter nos investigations au travers des dossiers concernant les contextes socio-économiques ${ }^{8}$ dans lesquels évolue l'enfant. La vie matérielle et l'environnement social ne sont pas considérés dans les dossiers comme 
des éléments permettant d'éclairer le placement. Ils illusionnent sur l'origine sociale des enfants protégés qui se perçoit bien souvent entre les lignes mais qui est rarement énoncée comme telle.

9 La dimension relationnelle dans un langage qui emprunte pour beaucoup à celui des professions «psy » est l'angle d'accès privilégié , depuis une trentaine d'années, pour raconter ces histoires de familles et ces histoires de placement. Par ailleurs, il convient d'interroger l'absence d'éléments contextuels sur le milieu d'origine. Souvent présenté en terme de carence, de défaillance, le milieu d'origine est bien évalué et jugé en fonction d'écarts à une norme sociale et ces écarts ne se construisent pas seulement sur le plan éducatif mais également sur le plan culturel, économique. Il va de soi que les intervenants sociaux connaissent ces situations, elles font l'objet d'enquêtes et de visites à domicile, elles sont discutées en réunion d'équipe. Pour autant, elles ne s'écrivent pas. "Si la plupart des familles sont dans le besoin, cette forme de dénuement n'est pratiquement jamais intégrée au sein d'un système interprétatif des difficultés (des enfants), simple indice d'un contexte banal et banalisé. ${ }^{10} \mathrm{~L}$ 'absence de cette dimension vise à exclure certains éléments de compréhension et donne bien souvent aux justifications autour du placement un caractère exclusivement relationnel.

Dans l'histoire du placement, se croisent acteurs collectifs et acteurs individuels, se croisent des intérêts partagés et des intérêts propres, se croisent l'histoire institutionnelle et l'histoire individuelle; chacun donne du sens à l'histoire mais n'attribue pas forcément le même sens à l'histoire.« Une question se pose alors : qui parle de qui ou mieux qui parle de quoi ? Alors que seul le sujet, moteur de sa vie, peut l'évoquer en terme de sens, il semble que ce soit les autres qui s'en chargent, et le sujet devient alors objet de savoir. Les jeunes femmes ont tout au long de l'intervention éducative été sensibles à des moments forts qui n'étaient pas toujours perçus par les acteurs sociaux, des zones de lumière ont surgi pour elles, elles n'étaient parfois que zones sombres pour eux. Des événements marquants pour les uns deviennent insignifiants pour les autres. Ces deux discours, différents certes, ont chacun leur propre vérité, il serait désolant que l'un des deux étouffe la voix de l'autre. ${ }^{11}$ Béatrice Koeppel dresse le constat de la construction unilatérale du sens, constat qui trouve écho encore aujourd'hui dans la manière dont se construit ce qui constitue une trace d'un passage dans l'institution: le dossier. Elle nous invite à réfléchir aux modes d'élaboration de la vérité de l'histoire ou des vérités de l'histoire selon les points de vue socialement construits et leurs articulations. Est-ce que des versions qui diffèrent peuvent co-exister ou est-ce qu'elles sont contraintes à se défier sans forcément bénéficier des mêmes armes pour "attaquer " ou "se défendre "? L'inégalité c'est, pour les uns, la possibilité de poser par écrit, d'y mettre le sceau de l'institution ${ }^{12}$, d'argumenter sur la base d'expertise professionnelle et pour les autres, celle de se faire entendre, celle de se faire voir.

\section{Incompréhension et colère}

11 Dans les extraits d'entretiens qui suivent, deux mères expriment leur colère face à la violence symbolique ressentie à la lecture des rapports qui les concernaient.

«Je ne les lis plus parce que la DASS marque que des conneries. Quoi qu'on dise nous, en tant que famille, ce n'est pas pris en compte. On nous fait bien comprendre qu'on est moins que rien, qu'on n'a rien à dire, que c'est eux qui décident. On doit 
faire exactement ce qu'ils disent et rien d'autre » [Mère de Gildas / 4 enfants placés en 1998]

«Tout au début du placement de Jordan, la deuxième année, on avait rendez-vous chez le juge et j'avais vu Mme Crenn [référente de l'Aide sociale à l'enfance] deux fois dans l'année. [...] Comment voulez-vous faire un compte rendu de la famille, les relations de Mme Potier [assistante familiale] et moi ? Comment voulez-vous faire un rapport sur une personne que vous voyez deux fois dans l'année? Donc devant le juge, Mme Crenn lit son compte rendu et à un moment je lui dis : «sur quoi vous vous basez? On ne se voit pas [...] Qu'est-ce que vous voulez aller juger quelqu'un sur deux fois dans l'année? » [Mère de Jordan / enfant placé en 2002]

Ces réactions parentales sont des coups d'épées dans l'eau si l'on considère l'histoire de placement du point de vue du dossier. Elles ne seront consignées dans le dossier qu'après une réécriture, une réinterprétation. Rares sont les dossiers où l'on trouve consignés des écrits de parents et ce n'est pas parce qu'ils ne sont pas conservés mais parce que les parents n'écrivent pas.

\section{«Vu le rapport, vu la note, etc. »}

Le père de Sophie ne comprend pas l'importance accordée à l'écrit (il reçoit beaucoup de courriers) aux dépens des rencontres physiques (mais peu de visites). Pendant l'entretien de recherche, il énumère le début d'un jugement « vu le rapport, vu la note, etc.» et s'étonne que tous ces documents aient été réalisés alors qu'il n'a pas été «vu ». Il n'y a pas de relation proportionnelle entre le nombre de documents écrits sur lui, sur ses enfants, sur sa famille et le nombre de rencontres. Le père de Sophie interroge le mode de communication utilisé. Quel droit de réponse a-t-il face à la somme de ces écrits? Est-ce que la réponse "orale » vaut face à l'écrit? Faut-il maîtriser le même langage et la même syntaxe pour se faire entendre?

Aux moyens inégaux pour agir pour, contre ou dans le placement, s'ajoute bien souvent une nébuleuse autour de ce qui motive le placement, autour de ce qui est reproché au parent, autour de ce qui va se passer pour l'enfant (la question des temporalités : où l'enfant va être placé ? Pour combien de temps ?) Dans certaines situations, le jugement est une hypothèse sur la situation familiale que le placement va chercher à confirmer ou infirmer, c'est un principe de précaution qui s'applique dans des configurations familiales qui cumulent différents types de risques pouvant engendrer un défaut de protection de la part des parents vis-à-vis de l'enfant. Le jugement est la trace « officielle» du placement et ne correspond plus, quelques années plus tard, à ce qui fait sens dans la séparation pour les différents acteurs.

D'un côté, le temps, l'enchaînement de nouveaux évènements, la maturité du regard porté sur la séparation, la meilleure connaissance de soi et de son environnement familial vont modifier le regard initialement porté sur le placement. De l'autre, le jeu des acteurs va influer fortement sur la perception des motifs de placement selon à la fois les sources d'informations à disposition et la manière dont chacun des acteurs se positionne vis-à-vis du placement.

16 L'exemple qui suit montre combien la perception du motif de placement s'intègre dans une dynamique d'expérience individuelle où chacun, selon où il se place et les informations qu'il perçoit, va plutôt mettre en avant tel ou tel aspect ayant pu motiver la séparation familiale. 
Anna, 20 ans, placée à l'âge de 8 ans.

17 Mr et Mme Corre ont trois enfants en commun. Anna a peu de souvenirs d'une vie de famille tous ensemble. Elle se souvient des absences de son père justifiées par son activité professionnelle et des difficultés de sa mère pour gérer seule ses trois enfants. Elle se souvient de fréquents déménagements, d'une mère qui peine à se trouver bien quelque part, de l'impatience qu'elle manifestait face à ses trois enfants.

« Elle le faisait et je me rappelle qu'après elle pleurait. Je me rappelle une fois elle m'avait tapé parce que j'avais fait un trou dans mon pyjama et dix minutes après elle était venue en disant : "prends maman dans tes bras" ».

Pour Anna, son placement a été motivé par des faits de maltraitance physique et par les problèmes maternels de surconsommation d'alcool : "J'ai été placée parce que ma mère a eu des soucis d'alcool et elle nous battait justement ». Anna pense que le placement est venu sanctionner les comportements de sa mère vis-à-vis de ses enfants alors que l'enchaînement des mesures et le contenu du premier jugement ne laisse pas percevoir ces motifs de placement.

19 Le jugement imposant le placement fait état d'une mesure d'investigation et d'orientation éducative (IOE) mise en place trois mois plus tôt mais le contenu de la décision manque de précision et le danger pour les enfants n'est pas caractérisé :

«La grande instabilité de Mme Corre sur tous les plans, instabilité à laquelle les enfants sont étroitement associés, la dégradation de sa situation dans ces derniers temps, les interrogations existant sur son mode de vie et sur le vécu des enfants lorsqu'ils sont avec elle, son incapacité à les protéger et à se remettre en question imposent le placement des enfants au service de l'Aide sociale à l'enfance dans un cadre judiciaire seul approprié en l'espèce pour garantir une protection suffisante des mineurs. » (Eléments du jugement en assistance éducative, 1995)

$20 \mathrm{Au}$ cours du placement, le jeune frère d'Anna accuse son père d'actes incestueux et ces révélations permettent d'ouvrir une enquête judiciaire. Anna est entendue et témoigne avoir subie les comportements déplacés d'un des compagnons de sa mère «il avait des gestes incestueux envers nous ». La procédure pénale aboutit à l'incarcération de l'ancien compagnon de la mère et innocente le père.

21 Les motifs initiaux du placement consignés dans le jugement sont interrogatifs mais se voient renforcés et étayés au cours du placement, à mesure que les enfants prennent de l'assurance pour dire et raconter. La mesure de protection prend du sens a posteriori sans pour autant recouvrir le sens qu'Anna lui a toujours donné.

Ce qu'Anna raconte sur les violences que lui infligeait sa mère n'apparait pas dans son dossier et n'apparait pas dans le discours de son assistante familiale : «c'est surtout de la négligence. La maman boit beaucoup donc est capable de délaisser ses enfants toute une nuit voire deux jours [...] Je ne pense pas qu'elle était battue par exemple, c'est plu, de la négligence.»

Le sens se construit sur l'expérience et ne peut pas s'appréhender seulement au travers des écrits. Pour Anna, cette expérience, c'est son vécu ; elle avait 8 ans quand elle a été placée et elle a pu élaborer sur son placement au moment de la séparation familiale. Pour son assistante familiale, cette expérience, c'est celle du quotidien partagé avec Anna, de ce qu'elle a pu percevoir dans ses comportements, dans ce que celle-ci a pu dire et de ce qu'elle a pu recueillir comme information auprès de l'institution par l'intermédiaire du référent de l'Aide sociale à l'enfance notamment. 


\section{Le rapport entretenu par l'enfant aux motifs de placement selon le type de parcours}

Anne Muxel distingue trois fonctions dans la mémoire familiale: une fonction de reviviscence (une forme de loyauté envers son expérience familiale), une fonction de transmission (l'inscription dans la continuité familiale), une fonction de réflexivité (dépassement de l'histoire familiale pour se construire son histoire). Chacun des types de parcours de placement que nous avons construits ("placés», "déplacés", "replacés ») mobilise dans le rapport entretenu à l'histoire familiale, une de ces fonctions.

\section{Les enfants placés, la construction de nouveaux supports avec le placement}

Les « enfants placés » sont ceux qui ont été placés jeunes (moins de 10 ans), qui ont connu un lieu de placement long et terminent (ou ont terminé) leur placement par ce lieu d'accueil. Intégré dans un autre fonctionnement familial que son milieu d'origine, assuré de la continuité de la prise en charge, investi des aspirations de sa famille d'accueil, l'enfant développe un parcours de placement qu'il juge de manière positive. Les parents existent mais les liens ont fluctué au cours du parcours et même s'ils persistent, les liens du quotidien ont pris le pas sur les liens du sang et les liens du droit, sans pour autant se substituer les uns aux autres. Le placement a permis la construction d'une nouvelle parenté, une parenté d'accueil comme l'ont montré les travaux d'Anne Cadoret ${ }^{13}$.

Les enfants placés ne cherchent plus à comprendre le motif du placement. Ils ont laissé de côté les motifs initiaux de la séparation familiale. Ils ne maîtrisent pas les éléments qui ont conduit au placement et ne cherchent pas à les maîtriser.

David, 13 ans, placé depuis l'âge de 2 ans.

David a peu de souvenirs de l'avant placement et s'est saisi de ce qu'on a bien voulu lui dire.

«Pourquoi tu as été placé?

- Je crois que déjà ma mère ne pouvait pas me gérer, déjà en plus, elle était jeune.

- Elle avait quel âge?

- 18 ans. Autrement je ne sais pas.

- Tu n'as jamais demandé?

- Si, sûrement mais on m'a jamais bien répondu vraiment pourquoi. Ça c'est $d u$ passé, j'ai pas trop envie de savoir. Si quelqu'un me le dit ce n'est pas grave mais ça ne m'intéresse pas. »

David a cherché à comprendre quand il était plus jeune, quand il avait encore des liens avec sa famille, la raison qui l'avait conduit à être placé. Mais ce que les adultes lui ont dit n'a pas constitué une réponse satisfaisante - voire suffisante... Le jugement ne nous donne pas davantage d'informations. Il évoque des carences multiples mais sans préciser de quelle nature sont ces carences :

«Melle Prigent ne respecte pas les engagements pris [...] Les éléments du dernier signalement mettent en évidence l'immaturité de Melle Prigent, son incapacité à appréhender les besoins de ses deux premiers enfants tant sur le plan matériel que sur le plan psychologique, et à $y$ répondre de manière 
satisfaisante. [...] Actuellement David et Jean sont en danger du fait des carences multiples relevées et leur placement à l'Aide sociale à l'enfance s'impose dans le cadre judiciaire afin de leur garantir une protection efficace." (Eléments du jugement en assistance éducative, 1996) des questions directes posées à la famille d'accueil, au référent, ou beaucoup plus rarement, à la famille d'origine, par l'attention portée à des discussions entre adultes, par ce qu'on se dit entre frères et sœurs et entre enfants placés dans le même lieu d'accueil. A un certain moment de son parcours - souvent au début - l'enfant va se fixer sur ce qui a amené le placement, se posant de multiples questions et ne trouvant auprès des adultes que des réponses partielles. Puis, le temps faisant et l'intégration dans un autre quotidien aidant, l'enfant va abandonner ou au moins mettre de côté l'idée de comprendre son histoire parce qu'elle n'est pas celle vécue par le collectif familial dans lequel il s'intègre. Les enfants arpentant ce type de parcours ne se présentent pas comme des enfants placés mais comme des enfants de la famille qui les accueille. En demandant au jeune de raconter son placement, on lui demande de revenir sur son histoire, sur les motifs de placement, sur les relations entretenues avec les parents... On le resitue dans son statut d'enfant placé. Toute la difficulté étant de comprendre ce qui aujourd'hui prend sens pour le jeune. A plusieurs reprises, nous avons notamment eu des difficultés à revenir sur la famille, parce que celle intériorisée par le jeune n'était pas celle consignée dans son dossier, dans son histoire généalogique. Nous avons souvent hésité à utiliser le terme famille d'accueil pour des enfants qui considéraient être dans leur famille, tout simplement. Revenir sur l'histoire du placement c'était aussi bousculer des positions qui s'étaient construites au cours des années sur l'expérience du quotidien et ramener l'enfant à son histoire c'était oublier ce qu'il avait réussi à construire.

Joris, 16 ans, placé à l'âge de 2 ans.

« Pourquoi tu as été placé au début?

- Parce que j'ai été maltraité avec mon père. Ça c'est ce qu'on m'a dit moi, je

m'en rappelle plus. Il buvait de l'alcool donc il nous maltraitait. Niveau salaire aussi, je crois qu'ils n'étaient pas vraiment au point donc j'ai été placé.

- On t'as dit quel type de maltraitance?

- Non.

- A quoi tu penses comme maltraitance? Des coups? Du délaissement?

- Je pense plus à des coups »

Joris n'éprouve pas le besoin de connaître l'histoire de sa situation, il sait des choses et fait des associations d'idées. Trop jeune au moment du placement pour maitriser ce qui l'a conduit en famille d'accueil, il ne connaît pas ses parents et l'histoire familiale. Jamais il n'a parlé du placement avec ses parents. Il s'est construit avec ce qu'on lui a dit, avec une certaine idée de ce qu'était sa vie familiale avant. Des représentations sans doute décalées par le poids des mots. Joris associe directement le terme maltraitance à des violences physiques alors que pourtant le jugement ne nous donne pas cette information.

Revue d'histoire de l'enfance « irrégulière », 11 | 2009 
«Attendu que la situation familiale est très fragile; qu'en effet, les problèmes financiers, la précarité matérielle, les difficultés du couple, l'autorité du père et l'état dépressif de la mère créent un climat très insécurisant pour les enfants ; que les parents adhèrent à la mesure de placement [...] » (Eléments du jugement en assistance éducative, 1993) au domicile familial. L'enfant investit a minima le placement parce que les conditions de son accueil ne sont pas pérennes ou quand elles le deviennent au terme de plusieurs changements, l'épuisement et la peur de créer des liens qui peuvent se voir fragilisés par une rupture font que l'enfant protégé par le placement se retrouve dans une situation où la sécurité n'est pas plus acquise que dans son milieu d'origine. De manière symbolique, l'enfant semble avoir perdu sa place. Michel Giraud ${ }^{16}$ parle de "déterritorialisation » de soi, des enfants en quête de liens et de lieux de vie improbables. 

plus présent que chez les "enfants placés " comme si la séparation était toujours d'actualité. «Rattachés » nulle part, ils ne semblent se reconnaître qu'au travers de la première séparation.

L'incompréhension de la prise en charge dans le cadre d'un placement se traduit par le retour systématique des enfants aux arguments du placement qui ne sont pas en concordance avec leur expérience et par la mise en échec des différents accompagnements mis en place. "Il en découle un étrange émiettement de l'enfant " pris en charge » par l'Aide sociale à l'enfance ; cet enfant dépossédé d'un passé qu'on lui cache et d'un avenir qu'il ne décide pas, cet enfant tiraillé entre une famille naturelle qu'il ne peut oublier et une famille d'accueil qu'il ne peut adopter; l'enfant déplacé de foyer en famille d'accueil, de famille d'accueil en établissement social, d'établissement social en établissement spécialisé - dix fois, vingt fois... Cet enfant aux mains de dizaines de personnes, administrations sociales, médecins, éducateurs, juges... Déraciné, coupé, tiraillé, morcelé. ${ }^{17}$

\section{Gildas, 15 ans, placé à l'âge de 6 ans}

\section{Le placement}

«Ma mère n'était pas prévenue et il $\mathrm{y}$ a des éducateurs qui sont venus nous chercher dans l'école [...] mes quatre sœurs et moi. Sans prévenir notre mère, ils nous ont emmenés dans une voiture et ils nous ont dit que notre mère, elle était malade. Donc après, on n'a pas eu de nouvelles de notre mère ou si, deux mois après, je crois. » [Gildas]

«Ils me les ont enlevés du jour au lendemain sans être prévenue. A midi, ils devaient rentrer à la maison pour manger comme d'habitude mais les enfants ne sont pas rentrés. Quand je suis venue à l'école pour savoir ce qui se passait, on m'a dit: «on n'est pas au courant». La gendarmerie est venue m'avertir que j'avais rendez-vous au tribunal à $14 \mathrm{~h}$ et arrivée au tribunal, on m'a dit : « vos enfants sont placés ». Je leur ai demandé des explications et on m'a donné aucune explication et en plus, on m'a dit: " vous n'êtes pas contente, si vous discutez c'est simple, on vous retire la garde définitivement de vos enfants, on vous enlève tous vos droits. " » [Mère de Gildas]

Ni Gildas, ni sa mère n'ont envisagé à l'époque une telle mesure. Ils s'étonnent de l'urgence avec laquelle la décision de placement a été prise.

Ce placement n'a pourtant pas été décidé de manière arbitraire par la justice et les services sociaux. Il s'était construit sur les relations entretenues entre les services compétents et Mme Ménez concernant la mesure d'AEMO ${ }^{18}$ judiciaire qui a précédé le placement où cette mère de famille était perçue comme faisant "barrage à toutes interventions extérieures jugées [par la mère] comme dangereuses». Le jugement précise donc que " pour des raisons de sécurité, les enfants seront placés avant l'audience, Mme devant être reçue l'après-midi ».

«Attendu qu'il ressort des rapports [d'IOE et d'AEMO] que Mme Ménez, au vécu extrêmement douloureux, est enfermée avec ses enfants dans un fonctionnement gravement pathologique; que l'intervention extérieure est rejetée ou disqualifiée ; que les enfants, à des degrés divers, sont contaminés par la pathologie maternelle et présentent tous des difficultés relationnelles et leur évolution est gravement compromise; ;...]qu'il ressort, en effet, du rapport d'expertise psychiatrique de la mère que "la famille est en grande détresse psychologique; qu'elle a besoin d'un 
solide soutien éducatif, psychologique, voire psychiatrique, mais que le dialogue avec Mme Ménez risque d'être très difficile dans la mesure où elle est à l'heure actuelle très opposante [...] " " (Eléments du jugement en assistance éducative, 1998). Les "enfants re-placés " sont ceux qui ont connu un enchaînement de mesures interrompu par un (ou des) retour(s) au domicile familial. L'enfant existe dans un entre-deux, entre chez ses parents et le placement, dans un mouvement d'aller-retour, dans une forme de garde alternée. L'enfant s'inscrit dans la continuité familiale et il est assuré que « son chez lui » est chez ses parents. La mesure de protection même si elle 
concerne nominativement le mineur est une négociation permanente entre les différents acteurs, un jeu à trois où enfant, parent(s) et professionnel(s) se reconnaissent un rôle, une place.

Les enfants re-placés sont ceux qui maîtrisent le mieux leur accueil et les raisons de celui-ci. Dans le jeu à trois, l'enfant est au moins consulté voire pleinement acteur si son âge le permet. La séparation familiale ne remet pas en cause la continuité familiale mais l'aménage. Le sentiment d'abandon que peut ressentir l' «enfant placé » ou « déplacé » n'est pas partagé par l' « enfant re-placé » qui sait qui est « sa » famille.

\section{Marie, 16 ans, placée à l'âge de 12 ans.}

Marie a connu deux placements. Le premier dans un cadre administratif, à la demande de ses parents. Le second dans un cadre judiciaire, à sa demande.

Elle connaît les difficultés de sa mère et l'indépendance de son père (liée à son activité professionnelle). Quand sa garde est confiée à son père, Marie se voit surtout confiée à sa belle-mère et la nature conflictuelle de leur relation sera le moteur principal de la demande de placement.

Le jugement annonce pour sa part des arguments plus évasifs mais retient le fait que la demande a été effectuée par la jeune alors âgée de 14 ans :

« Marie a demandé à bénéficier de stabilité et d'un cadre plus rassurant ce dont elle a besoin [...] Ordonnons le placement de Marie [...]» (Eléments du jugement en assistance éducative, 2005).

Marie n'a pas eu de difficultés à se situer entre «sa » famille et son lieu d'accueil. Le placement est ici vécu de la même manière que pourrait l'être un internat scolaire à la différence près qu'il faut ici pouvoir argumenter la nécessité d'une protection :

«Moi, j'étais aussi bien ici. Mais comme on me dit, il n'y a pas d'argument pour que je reste placée [...] Et il y a d'autres enfants qui ont une situation pire que la mienne qui ont besoin d'être placés. Moi ce n'est pas du tout pareil parce que j'ai ma famille qui peut s'occuper de moi et je ne suis pas en danger avec mes parents donc il n'y a aucune raison que je reste placée. »

\section{Dylan, 14 ans, placé à l'âge de 11 ans.}

Le placement de Dylan et de sa jeune sœur est le fruit d'une négociation active entre le père, les services sociaux et la justice. Des antécédents existaient: un suivi social important et le placement difficile des deux frères aînés de Dylan près de quinze ans plus tôt. Le père de Dylan était méfiant vis-à-vis des services sociaux et savait ce qu'il souhaitait : "un accueil ponctuel, quelques jours par semaine et un week-end sur deux » mais craignait de ne pas être écouté. Il considère comme une chance d'avoir rencontré cette juge, une juge qui a pris le temps d'écouter ce qu'il avait à dire et ses souhaits mais, rajoute-il, «à sa place, le commun des mortels aurait été blousé ». Il a bien conscience que la frontière entre les différentes mesures est très poreuse et que l'appréciation du juge dépend de ce que les travailleurs sociaux peuvent raconter si les parents n'ont pas la force d'exprimer eux-mêmes leur difficultés (et préfèrent se laisser porter par l'appréciation professionnelle des travailleurs sociaux "qui savent mieux qu'eux»). Un placement ou un accueil provisoire, ou est la différence ? Pour Mr Bégard, Dylan n'est pas placé en famille d'accueil mais gardé par une «nounou » qui a su "l'adopter », «Dylan est comme à la maison là-bas ». 
Dylan a compris ce placement : «mon père, il travaillait beaucoup et on est quatre enfants et ma mère est décédée ». Il n'avait pas envisagé de pouvoir vivre ailleurs que chez son père mais il était d'accord. Les relations dans la fratrie s'étaient dégradées et Dylan avait besoin de souffler. Trop jeune et trop seul pour se défendre et se protéger, il a très vite compris que cette famille d'accueil c'était son «joker». Dylan a vécu et vit positivement son placement «ça aide beaucoup [...] pour grandir, pour que ça se passe mieux chez moi, des gens dans la semaine pour aller dans ma famille d'accueil ou chez mon père "

La maîtrise du parcours se double de la maîtrise de l'histoire familiale ou du moins, de l'envie de la maitriser. Ces enfants savent où ils s'inscrivent, ont conscience des difficultés ou des carences de leur milieu d'origine et se serve du placement comme d'une possibilité pour atténuer ces difficultés. La notion de « joker » évoquée par Dylan illustre la distribution du jeu, entre milieu d'origine, milieu d'accueil et enfant. Chacun a des cartes en main mais personne n'a toutes les cartes et l'enfant a la possibilité de lancer ou de suspendre le jeu. Les règles sont élaborées par l'institution mais la partie ne peut exister que par le jeu des acteurs. Si le rapport institution/parent peut s'apparenter à un rapport dominant/dominé, on aurait tort de le réduire à cette seule dimension. Tous les parents reconnaissent l'autorité de l'institution. Pour autant cette autorité n'est pas toujours vécue comme totalitaire. Des marges de manœuvre existent aussi pour les parents et certains professionnels sont également prêts à les négocier, reconnaissant aux parents des compétences primordiales dans l'orientation à donner au parcours de placement de l'enfant. La parentalité, en tant que fonction, se partage et l'enfant est un acteur à part entière dans le partage de ces compétences parentales.

\section{Un parcours évalué, consigné}

Les écrits qui se trouvent consignés dans les dossiers des enfants placés ont plusieurs fonctions : l'évaluation (en amont de la mesure, en cours de mesure), l'information/la communication (entre les différents services en charge de l'enfant, entre parents et institution), la mémoire (pour l'institution, pour les enfants devenus adultes ou sortis du dispositif, pour les parents).

Evalués par des professionnels de la Protection de l'enfance, les parents et les enfants se reconnaissent rarement dans le portrait dressé sur eux, sur leur famille. De cette histoire familiale racontée à maintes reprises dans le parcours de placement, à plusieurs professionnels, ils peinent à se démarquer.

\section{« lorsque l'on creuse la plaie, on l'agrandit ! » ${ }^{20}$}

61 Dans les trois témoignages qui suivent, enfant et parents expliquent leur épuisement à devoir se raconter à des professionnels différents.

«Ce qui m'a gêné, c'est d'avoir plein de référentes. Ça changeait tout le temps, pour l'enfant c'est plus facile d'être habitué à une référente. Parce qu'on se confie déjà, le fait de parler de son histoire, s'il faut le refaire tous les ans, personnellement ça me gêne. J'en ai eu au moins 4 ou 5 en l'espace de 5 ans au début. » [Anna, 20 ans, placée à l'âge de 8 ans]

«Le problème du CDAS [centre départemental d'action sociale] c'est pas que c'est mal fait, c'est pas critiqué... On a eu une personne donc un éducateur qu'est venu, ça a duré 15 jours puis il a été muté, après on a eu un autre, ça a duré peu de temps puis il est muté après il y en a eu un autre... Et, stop ! Je ne suis pas là pour raconter 
ma vie 50 fois donc, c'est vrai quelque part c'est un truc que je ne comprend pas chez eux. On a eu des soucis avec Jordan, je vous explique même pas, trop à faire à trop de personnes différentes et on recommence, et on recommence... Où vous voulez qu'il y ait un équilibre quelque part? [...] Il faut que l'enfant arrive à avoir confiance dans la personne avec qui il a des relations, avec qui il a à faire. Faut qu'ils instaurent une relation si au bout de tant de temps, on change non, stop ! On ne recommence pas à chaque fois à zéro. [...] C'est bien qu'il ait eu la même famille d'accueil parce que chez eux ça se passe bien parce que en plus, s'il fallait qu'il change non, stop! Franchement je le récupère parce que c'est déjà contraignant d'avoir à faire à beaucoup de gens, parce qu'on a vu le psychologue, on a vu... il y a eu aussi une enquête de faite tout au début du placement [...] C'est tout un cheminement. Et si, en plus, on voit des gens extérieurs plus la famille plus la référente plus le juge et stop! [...] Au début, c'est dur c'est pas évident d'aller raconter... » [Mère de Jordan / enfant placé en 2002]

« [...] dans les services ici il y a beaucoup de changement et à chaque fois, il faut se répéter, c'est ça qui est pénible. Ça rouvre des blessures et des choses dont on ne veut plus parler. Donc il faut se répéter, comme avec vous actuellement. [...] On est des humains, on n'est pas des machines. " [Parents de Antoine / enfant placé en 2005]

Pierrine Robin ${ }^{21}$ en partant du point de vue de l'enfant sur ces évaluations questionne leur nature elle parle d'évaluation proximale où les professionnels qui évaluent ne sont pas forcément ceux qui connaissent le mieux l'enfant ; d'évaluation endoformative avec un renvoi systématique et récurrent à l'histoire familiale; et d'évaluation unilatérale dans le sens où les attentes s'adressent de manière quasi-exclusive à la famille et sont rarement interrogés les moyens mis en place par les acteurs administratifs pour répondre aux objectifs de la mesure.

Mais au-delà de la mesure, les enfants dans leur prise en charge quotidienne profitent des marges de manœuvre qui leur restent, évaluent eux-mêmes leur situation et construisent leur parcours. «Essentiellement opérationnelles, les intimités infusent dans l'institution un peu de leurs propres fins, une réflexivité stratégique ou une rétrospective personnelle favorable, s'approprient ponctuellement une marge de jeu, luttent pour arracher un bref moment secret, sauvegardent un geste du regard, ou protègent, ne serait-ce qu'un instant, la confidence d'un échange. Se glissent aussi des changements dont les individus sont amenés à prêter sens à leurs conduites, à leurs devoirs, à leurs plaisirs et à leur résistance. La bataille est sur chaque instant, fragile et menacée, toujours minée et négociée avec le personnel. Les intimités grignotées, réécrites, écornées incitent à bricoler la présentation de soi en tendant, selon les occasions et les opportunités, la corde du crédit ou du discrédit ou en jouant sur les incivilités permises et les nécessaires civilités. $»^{22}$

Le rapport à l'histoire du placement, à la mémoire familiale s'élabore en fonction de ce qui se construit et se déconstruit dans les différentes sphères sociales auxquelles participe ou participait l'enfant. Rupture ou continuité, oubli ou présence de l'histoire familiale, le parcours se conjugue au travers du jeu des acteurs (parent, fratrie, enfant, famille d'accueil, travailleurs sociaux, etc.) et des conditions objectives (âge de l'enfant, modalité d'accueil de la fratrie, type de mesure, motifs de la décision, etc.) dans lesquelles se déroule le placement.

65 L'expérience du placement racontée par l'enfant, par les parents ou par les professionnels du quotidien de la prise en charge nous révèle ce que les écrits nous taisent, c'est-à-dire des points de vue d'acteurs impliqués dans le dispositif et pas seulement celui des évaluateurs. On comprend dès lors la déception des parents et des 
enfants quand ils accèdent à leur dossier. Ils sont face à une histoire réécrite qui s'appuie sur la connaissance partielle d'un unique angle de vue, celui d'une institution qui rend des comptes à elle-même. Alors que pourtant, la construction du sens de l'histoire du placement s'adosse à des expériences subjectives et objectives d'acteurs impliqués dans le milieu d'origine, dans le milieu d'accueil ou dans les deux. «A la jointure du symbolique et de l'empirique, la parole privée témoigne de ce que le sens ne vient pas seulement d'en haut, mais qu'il se produit aussi « en bas » ${ }^{23}$.

\section{NOTES}

1. Jugement stipulant la mesure de placement ou document administratif d'accueil provisoire.

2. Emilie Potin, Annick Madec (Dir. Scientifique), Parcours de placement. Du simple lieu d'accueil à la négociation d'une place dans une "autre" famille, rapport de recherche remis au conseil général du Finistère, Atelier de Recherche Sociologique / Université de Bretagne Occidentale, novembre 2007.

3. Pour des raisons de respect de la vie privée, les noms et les prénoms des personnes qui apparaissent dans les extraits d'entretiens qui suivent sont des pseudonymes.

4. «Les archives sont l'ensemble des documents, quels que soient leur date, leur forme et leur support matériel, produits ou reçus par toute personne physique ou morale, et par tout service ou organisme public ou privé, dans l'exercice de leur activité. La conservation de ces documents est organisée dans l'intérêt public tant pour les besoins de la gestion et de la justification des droits des personnes physiques ou morales, publiques ou privées, que pour la documentation historique de la recherche. » Art $1^{\mathrm{er}}$ loi ${ }^{\circ} 79-18$ du 3 janvier 1979 sur les archives.

5. Frédéric Vabre, L'action publique contre la maltraitance des enfants. Eléments pour une approche de sociologie politique comparative sur les cas français et espagnol, Dossiers d'étude ${ }^{\circ} 65$, ENS de Cachan, CNAF, février 2005.

6. Jean-Claude Passeron, Le raisonnement sociologique. L'espace non-popperien du raisonnement naturel, Paris, Nathan, 1991.

7. Les dossiers sont archivés par fratrie et dans certains dossiers, ceux constitués avant 1994, se mêlent à l'histoire de placement des enfants, celle des parents quand ils ont été placés dans le département.

8. Notre enquête interrogeait notamment les conditions de logement dans la famille d'origine et dans la famille d'accueil mais la faiblesse des réponses (139 répondants sur 341 interrogés concernant le logement familial et 60 concernant le logement de la première famille d'accueil) ne nous a pas permis de traiter cette question.

9. Robert Castel, Jean-François Le Cerf, «Le phénomène " psy » et la société française », Le débat, $1980, n^{\circ} 1-2-3$.

10. Michel Giraud, «Le travail psychosocial des enfants placés », Déviance et Société, 2005/4, Vol. 29, p. 463-485, p. 467.

11. Béatrice Koeppel, «On m'a placée un peu partout », Enquêtes et recherches, Vaucresson, 1977, p. 274 ; voir aussi Marguerite B. Une Jeune fille en maison de correction, Hachette, 1987.

12. "[...] dans une biographie reconstituée "sous contrainte", celle habituellement exigée aux différents guichets sociaux, l'institution a une force d'évidence, édicte d'exhaustives règles de papier, emblème des 
conditions et des positions. »; Jean-FrançoisLae, Bruno Proth, "Les territoires de l'intimité, protection et sanction ", Ethnologie française, Paris, PUF, 2002/1, p. 5-10, p. 8.

13. Anne Cadoret, Parenté plurielle. Anthropologie du placement familial, Paris, L'Harmattan, 1995.

14. Ibid., p. 147.

15. "Je me sens chez moi ici. Alors qu'avec mes parents je pense que ça n'aurait pas été pareil. Je sais pas comment le dire. Je suis mieux ici [...] Quand je parle comme ça c'est "maman " "papa » mais moi je sais qui c'est mes vrais parents, ça reste ma famille d'accueil mais je les considère comme mes parents. » [Joris, 16 ans, placé depuis 13 ans dans la même famille d'accueil].

"Au début, ce n'était pas encore ma vraie famille [David parle de sa famille d'accueil],je ne les connaissais pas beaucoup mais après... maintenant c'est ma famille." [David, 13 ans, placé depuis 11 ans dans la même famille d'accueil.]

Joris utilise «vrais parents» pour qualifier sa famille d'origine tandis que David l'utilise concernant sa famille d'accueil. Il parle pourtant du même processus, d'une même place accordée à leurs deux catégories de parents mais les expressions se confondent et manquent pour qualifier ces configurations familiales.

16. Michel Giraud, Op. cit.

17. Pierre Verdier, L'enfant en miettes. L'aide sociale à l'enfance : bilan et perspectives, Dunod, 1978, p. 3.

18. Action Educative en Milieu Ouvert

19. Anne Muxel, Individu et mémoire familiale, Hachette Littératures, Paris, 2001, p. 24.

20. Michèle Siguier, Droits de regards. L'enquête sociale dans la protection judiciaire de la jeunesse. Evolution depuis le XIX ème siècle. Valeurs de références actuelles. Incertitudes de son emploi futur, Toulouse, Erès, 1986, p. 109.

21. Pierrine Robin, "L'évaluation de la maltraitance en tension", Séminaire de l'observatoire national de l'enfance en danger, 6/02/09.

22. Jean-François Lae, Bruno Proth, op. cit., p. 5-10, p. 8.

23. Olivier Schwartz, "Le baroque des biographies ", Les Cahiers de Philosophie, n¹0, 1990, p. 173-183, p. 176.

\section{RÉSUMÉS}

Joris, Anna, Stéphane, Astrid... sont confiés dans le cadre d'un placement à l'Aide sociale à l'enfance. Ils partagent tous cette expérience d'« enfants placés » mais ils entretiennent chacun un rapport singulier à l'histoire de leur placement. L'auteure cherche au travers des parcours de placement à observer les écarts, les marges entre la manière dont les enfants peuvent se percevoir et envisager leur histoire familiale et la manière dont leur histoire, leur évolution est consignée, écrite, mise en scène dans les écrits institutionnels.

\section{Between childhood experience and institutional records: stories of placement}

Joris, Anna, Stéphane, Astrid... are assigned in the context of a placement by Children's Social Welfare. They all share the experience of "foster children" but they each maintain a unique relationship in the story of their placement. The author seeks to travel through placement to take note of the gaps, the differences between the way in which these children might perceive and envisage their family history and the manner in which their history and development is recorded, written and staged in institutional writings. 
INDEX

Index géographique : France

Mots-clés : écrits institutionnels, histoire familiale, placement, récit de vie Index chronologique : XXIème siècle

\section{AUTEUR}

\section{EMILIE POTIN}

Ingénieur d'études doctorante à l'Université de Bretagne Occidentale 